\title{
Glycemic variability is associated with poor outcomes in pediatric hematopoietic stem cell transplant patients
}

\author{
Jenna Sopfe ${ }^{1}$, Kristen Campbell ${ }^{1}$, AMY Keating ${ }^{1}$, Laura Pyle ${ }^{1}$, Arthur Liu ${ }^{1}$, Michael \\ Verneris $^{1}$, Roger Giller ${ }^{1}$, and Gregory Forlenza ${ }^{1}$ \\ ${ }^{1}$ University of Colorado Denver School of Medicine
}

May 14, 2020

\begin{abstract}
Background: Among pediatric hematopoietic stem cell transplant (HSCT) recipients, abnormal glycemic control is shown to be associated with increased risk of transplant-related mortality, death from any cause, risk of infection, increased hospitalized and intensive care days. Independent effects of higher glycemic variability, a component of glycemic control, have not been described. This study aimed to characterize risk factors for, and consequences of, higher glycemic variability in HSCT patients. Procedure: Medical records for a cohort of 344 patients, age 0-30 years, who underwent first HSCT from 2007-2016 at Children's Hospital Colorado were retrospectively reviewed. Glucose coefficients of variation (CV) were analyzed for HSCT days -14 to 0 and 0-30, and patients were assessed for potential risk factors and outcomes. Results: Roughly one third of patients had pre-HSCT and day 0-30 glucose CV above the reported healthy adult range. Independent of HSCT type, doubling of pre-HSCT glucose CV was associated with a 4.91-fold (95\% CI 1.40-17.24) increased hazard of infection, as well as increased risk for intensive care hospitalization for allogenic HSCT patients. Multivariable analysis demonstrated that allogeneic HSCT patients had a 1.40and 1.38-fold (95\% CI 0.98-1.99 and 1.00-1.91) increased hazard of death for every doubling of pre-HSCT and Day 0-30 glucose $\mathrm{CV}$, respectively. Conclusions: Just as with higher mean glucose, higher glycemic variability in the pediatric HSCT population is independently associated with significantly increased morbidity. Additional research is required to evaluate the utility of glucose control to mitigate these relationships and improve HSCT outcomes.
\end{abstract}

\section{Patients}

An existing HSCT program database was used to identify all pediatric/AYA patients who underwent first HSCT at a single academic children's hospital from 2007-2016. Demographic and clinical data were extracted from this database and electronic health records through March 31, 2017 via research informatics and manual review. Inclusion criteria were: 1) age 0-30 at time of transplant, and 2) allogeneic or autologous HSCT recipient at Children's Hospital Colorado between January 1, 2007 and July 31, 2016. Exclusion criteria were: 1) pre-existing diabetes mellitus, 2) insulin requirement within the two weeks prior to transplant, and 3 ) inadequate blood glucose data $(<20$ measurements total or $<2$ in a given period (pre-HSCT and post-HSCT day 0-30)). Of 351 patients identified, the primary analyses included 344 patients. Four additional patients were excluded only from pre-HSCT analyses due to too few glucose measurements pre-HSCT ( $\mathrm{n}=340$ for these analyses). This study was approved by the University of Colorado Institutional Review Board.

Exposure and outcomes definitions

While debate exists among clinicians and statisticians about the best representation of GV, CV has begun to emerge as the preferred metric due to its advantage of being relative to mean glucose and thus more descriptive of hypoglycemic excursions than SD, which is largely only reflective of hyperglycemic excursions. ${ }^{4-7}$ Therefore, CV was used to measure GV for each subject using laboratory-measured serum glucose values over pre-transplant (day -14 to 0) and post-HSCT (days 0-30) intervals to evaluate for HSCT-related stress 
$\mathrm{GV}$ and its effects. Because glucose $\mathrm{CV}$ is meant to reflect nonfasting glucose over an extended period, all glucose values were included, exceptglucose values within one hour of a previous glucose value were censored to reduce oversampling. Evaluation of potential outliers did not significantly impact results. Whole blood glucose measurements were typically performed daily in the early morning (between 12 and 3 A.M.) during hospital admission, and typically one to three daytime measurements per week after discharge. Because natural glucose CV categories are not clearly defined, we analyzed glucose CV continuously for all models. However, for descriptive purposes, glucose CV was categorized into four groups approximating published nonfasting CV ranges in various populations. The groups are: healthy adults (Grade $1,<20 \%$ ), adults who are obese or receive aggressive oral therapy for type 2 diabetes (Grade 2, [?]20 and $<27 \%$ ), adults with type 1 or 2 diabetes that is well-controlled with insulin (Grade 3, [?]27 and <36\%) and adults with poorly controlled type 1 diabetes (Grade 4, [?]36\%).5,8

Post-HSCT outcomes included time to: death from any cause, transplant-related mortality (TRM), diagnosis of severe GVHD (defined as acute grades III-IV or chronic) ${ }^{9,10}$, clinically significant infection in the first 100 days, and ICU admission in the first 100 days post-HSCT. Clinically significant infections were defined by hospitalization for a positive microbiology result or diagnostic/problem list code. Infections were further categorized by serious bacterial infection (SBI), viremia/viruria, or invasive fungal infection. Serious bacterial infections included infections such as bacteremia, bacterial peritonitis, meningitis, and pneumonia with hypoxemia. To be classified as clinically significant for viremia/viruria, we required both positive high viral load (of Ebstein Barr virus, Human herpes virus 6, adenovirus, cytomegalovirus, and BK virus) and symptoms requiring hospitalization and treatment, when available. To reduce the risk of misclassification bias, subjective outcomes (infection, GVHD) were reviewed by at least one study physician; any unclear outcomes were classified by 1-3 additional study physicians.

A list of exposure variables/covariates and their definitions is provided in the appendix (Table S1). Data were censored at the time of any recurrence/progression of primary disease, graft failure, or second HSCT. Because of the nature of HSCT care, there was not a notable amount of missing data or loss to follow-up.

\section{Statistical analysis}

For all analyses, SAS v9.4 and R v 3.6.1 and a significance level of 0.05 was used. Descriptive statistics included median and percentiles (minimum, $25^{\text {th }}$ percentile, $75^{\text {th }}$ percentile, maximum) for continuous variables, and counts and percentages for categorical variables. For all inferential analyses, glucose CV was $\log$ transformed due to non-normal distribution. Base 2 was chosen for ease of interpretation and the estimates were back transformed.

Linear regression was used to test for associations between potential risk factors for increased glucose CV at each time intervals. Potential predictors included: age, BMI category, primary diagnosis, conditioning regimen, and pre-transplant steroids, asparaginase, insulin, and radiation. For the post-HSCT glucose CV model, additional predictors were considered: HSCT type, and post-HSCT steroids, radiation, and total parenteral nutrition (TPN). The final models were chosen based on the lowest Akaike information criterion (AIC). An additional multivariable linear regression model evaluated the effects of post-HSCT immunosuppressive agents (sirolimus, tacrolimus, cyclosporine, methotrexate) on post-HSCT glucose CV.

Associations between glucose CV and time-to-HSCT outcomes were examined using Cox proportional hazards models. Glucose values were censored at the time of the event. For time-to-infection, ICU and GVHD analyses, death was treated as a competing risk. For TRM, death due to other causes was treated as a competing risk. Associations between pre-HSCT and day 0-30 glucose CV and both time to death and TRM were adjusted for HSCT type, severe GVHD diagnosis, and the need for post-HSCT steroids. The model evaluating day 0-30 glucose CV and GVHD excluded patients with GVHD in the first 30 days $(\mathrm{n}=9)$ and adjusted for steroids received post-HSCT but pre-GVHD diagnosis.

Because infections and ICU stays frequently occurred soon after HSCT (the median days to infection was 19 (interquartile range [IQR] 10.5-32.5)), only pre-HSCT glucose CV could be evaluated for an association with time to these events. Similarly, due to the short follow-up window post-HSCT and pre-infection/ICU 
stay, we were unable to adjust for steroid exposure, but we did adjust for HSCT type. Associations between pre-HSCT glucose CV and infection subtypes were evaluated using logistic regression, as patients could have had multiple types of infections, requiring significant censoring for each infection subtype. Viremia was evaluated only in patients who underwent allogeneic HSCT, as these were the only patients who underwent routine viral surveillance testing. Interactions between glucose CV and transplant type as well as glucose $\mathrm{CV}$ and steroids were tested for in all models and included if $\mathrm{p}<0.10$ for the interaction term.

To assess for the effect of possible outliers in glucose values, all analyses were re-run without glucose values $>500 \mathrm{mg} / \mathrm{dL}$. This did not significantly change any results, so is not reported.

Results

\section{Patient Characteristics}

Among the 344 patients included in the full cohort, median age at transplant was 8.0 years (IQR (3.015.0 ) and $59 \%$ were male (Table 1 ). Of all transplants, $65 \%$ were allogeneic and the primary indication for transplant was malignancy in $75 \%$ of patients.

Glycemic variability

There was a median of 37 glucose measurements from day -14 to day 30 per subject (IQR 32-40), with a median of 8 pre-HSCT (IQR 8-8), and 30 in days 0-30 (IQR 24-31). The median glucose CV was $17.8 \%$ (IQR 11.2-24.4\%) pre-HSCT and 15.5\% (IQR 11.6-24.2\%) post-HSCT. While no normal glucose CV ranges have been described in pediatric patients, $38 \%$ of patients pre-HSCT and $33 \%$ of patients in day 0-30 had a CV above the reported healthy adult range (Table S2). ${ }^{8}$ Figure 1 demonstrates CV distributions by time interval and glucose CV grade.

Table 3 demonstrates multivariable models to assess for risk factors associated with glucose CV. Prior asparaginase exposure and non-malignant diagnosis were associated with higher pre-HSCT CV, whereas postHSCT glucose CV was increased with pre-HSCT radiation to the pancreas (excluding total body irradiation [TBI]), prior insulin therapy, allogeneic HSCT, day 0-30 steroid exposure, and post-HSCT TPN. Overweight BMI was associated with decreased glucose post-HSCT CV. Additionally, among patients who underwent allogeneic transplant, GVHD prophylaxis with tacrolimus was associated with increased glucose CV after adjusting for exposure to other immunosuppressive agents.

\section{Overall Survival and Treatment-Related Mortality}

The median duration of follow-up for surviving patients was 2.9 years (IQR 1.3-4.8) years. Death occurred in $100(29.1 \%)$ patients, with 60 dying from the primary disease, 36 from a complication, and four from another/unknown cause. Of patients who died post-HSCT, the median days from HSCT to death was 264 (IQR 135-500).

The relationship between glucose $\mathrm{CV}$ and risk of death was dependent on HSCT type ( $\mathrm{p}=0.079$ pre-HSCT and $\mathrm{p}=0.024$ post-HSCT). After adjusting for post-HSCT steroids and GVHD, doubling of day 0-30 glucose CV was associated with a $1.38(95 \%$ CI: 0.996-1.91 p=0.053) fold increased risk of death among allogeneic HSCT patients, versus a $0.75(95 \%$ CI: $0.48-1.15 ; \mathrm{p}=0.18)$ fold increased risk among autologous HSCT patients (Table 3, Figure 2). Glucose CV was not associated with treatment-related mortality (TRM) (Table 3, Figure 2).

\section{Infection}

In the first 100 days post-transplant, 195 (57\%) patients had at least one documented infection; 80 (23.3\%) had a SBI, $153(44 \%)$ had viremia/viruria, and $14(4 \%)$ had an invasive fungal infection. The median days to infection was 19 (IQR 10.5-32.5).

After adjusting for HSCT type, there was a 4.91 (95\% CI 1.40-17.24; $\mathrm{p}=0.013$ ) fold increased hazard of infection with every doubling of pre-HSCT glucose CV (Table 3, Figure 3). While there was no association between pre-HSCT glucose CV and odds of SBI or fungal infection in the first 100 days, among allogeneic 
HSCT patients, the odds of viremia was 1.77 (95\% 1.23-2.56; $\mathrm{p}=0.002)$ fold higher with each doubling of preHSCT glucose CV. Because $71 \%$ of infections occurred in the first 30 days, it was not possible to evaluate for an association between day 0-30 post-HSCT glucose CV and time-to-infection due to overlapping intervals.

\section{GVHD}

Of 225 patients receiving allogeneic transplants, 50 (22.2\%) developed severe GVHD (median days to diagnosis 74 (IQR 37-208)) (Table 3). Neither pre-HSCT nor day 0-30 GV were associated with time-to-GVHD in multivariable analysis.

\section{ICU Hospitalization}

Intensive care hospitalization in the first 100 days was necessary for $75(22 \%)$ patients, with a median of 29 days (IQR 11-54) between HSCT and ICU hospitalization. For allogeneic HSCT patients, there was a 1.65 (95\% CI 1.15-2.35; $\mathrm{p}=0.006)$ fold increased risk of ICU hospitalization for every doubling of pre-HSCT glucose CV. There was no association among autologous HSCT patients. Because $53 \%$ of patients requiring ICU hospitalization did so before day 30, associations between day 0-30 variability and time-to-ICU could not be evaluated.

\section{Discussion}

This retrospective cohort study demonstrates that GV is common in pediatric patients undergoing HSCT, and that it is associated with increased risk of infection and critical illness. These associations are consistent with research describing malglycemia as a whole, both in adult and pediatric HSCT patients. ${ }^{1-3}$ Associations between GV and mortality were less clear.

Roughly one third of patients both pre- and post-HSCT had glucose CVs that were in the range of adults who are either obese or have diabetes, with a striking 8-10\% of pediatric patients having glucose CVs above the proposed cutoff for poor control in patients with type 1 diabetes. ${ }^{5}$ This suggests that the physiologic stress or iatrogenic exposures prior to and during HSCT, such as pre-HSCT asparaginase, radiation and preparative chemotherapy, or post-HSCT intravenous fluids, TPN, immunosuppressive agents, and steroids, appears to lead to abnormal insulin secretion or sensitivity, resulting in pediatric HSCT patients experiencing a pathologic level of GV.

In the pre-HSCT period, non-malignant diagnosis and prior treatment with asparaginase were associated with increased glucose CV. Asparaginase has known associations with pancreatitis and hyperglycemia, so this finding is not surprising. ${ }^{11-13}$ However, the reason for an association between non-malignant HSCT indications and higher glucose CV is unclear, as no patients in this group had disorders with known associations with glucose intolerance, and no patients had existing diabetes mellitus. ${ }^{14}$ It is possible that patients with malignant underlying diagnoses were more tolerant of the stress associated with HSCT preparation due to their prior treatment with chemotherapy, while for nonmalignant HSCT patients, HSCT preparation may have been more disruptive. While our prior research evaluating risk factors for pre-HSCT malglycemia identified underlying malignant diagnosis and adolescent age as risk factors, these were not associated with pre-HSCT glucose CV; thus, these findings were likely driven by hyperglycemia and not variability. ${ }^{3}$ Additionally, several other hypothesized risk factors, such as being overweight/obese, having prior steroid exposure, or having prior insulin needs, were not significant risk factors for increased pre-HSCT glucose CV. This may suggest that stress and exposures associated with HSCT result in these patients having similar glucose tolerance to an obese adult, regardless of BMI. The number of obese/overweight patients was small, so further studies to confirm this relationship are needed.

Consistent with prior literature on malglycemia or hyperglycemia, post-HSCT glucose CV was increased for patients who underwent allogeneic HSCT or who were treated with post-HSCT steroids or TPN. . $^{1,3,15-19}$ Prior insulin needs and any pre-HSCT radiation to the pancreas were associated with post-HSCT but not pre-HSCT increased glucose CV, suggesting that either the stress/exposures of undergoing a conditioning regimen levels out these factors pre-HSCT, or that post-HSCT, patients are more sensitive to their prior exposures. 
The high prevalence of elevated glucose $\mathrm{CV}$ in this study, and risk factors for it, are notable because increased glucose CV was associated with adverse HSCT outcomes. Specifically, there was nearly a 5 -fold increased risk of infection with doubling of pre-HSCT glucose CV, after adjusting for HSCT type. As can be visualized in Figure 1, median glucose CVs spanned a wide range, so differences in glucose CV of this magnitude (for example from $15 \%$ to $30 \%$ ) are not an uncommon occurrence. Fortunately, there was not a similar association for patients who underwent autologous HSCT, which may be related to lower risk of early death.

While glucose CV was associated with viremia among allogeneic HSCT patients, our analysis did not demonstrate significant associations with SBI or fungal infection. This could be due to the confounding factor of the potential for multiple infections of different subtypes and inability to explore time-to-infection subtype, or due to the low number of fungal infections in our cohort. While potential underlying pathophysiology is detailed more below, future studies are needed to explore the mechanisms underlying the association between GV and viremia, including an evaluation of additional factors such as conditioning regimens (e.g. T-cell depletion), and lymphocyte function and microenvironment. As may be expected given the associations between GV and risk of infection, there was also an association between glucose CV and ICU hospitalization, but only among allogeneic HSCT patients. While we did not evaluate for an association between GV and relapse based on lack of association between malglycemia and relapse in our prior study, future studies may evaluate this specifically, and should also consider the association between GV and rarer events, such as graft failure, sinusoidal obstructive syndrome, and thrombotic microangiopathy. This study was not able to evaluate the latter two of these events not only because of anticipated low numbers of events, but because diagnosis and treatment of these events changed drastically over the course of this observation period. Of note, in addition to adjusting for treatment with steroids, HSCT type and other potential confounders when able, for all analyses, glucose values after the time of the event were censored. By doing so, any GV preceded the associated event. demonstrating temporality, which is critical in considering potential causation.

While the underlying pathophysiology of the association between hyperglycemia and adverse outcomes is more clearly hypothesized to be related to known immunologic effects such as increased inflammation and impaired leukocyte function, the possible pathophysiologic relationship between GV and outcomes is less well described. ${ }^{1,15,20-23}$ However, several studies have demonstrated associations between wide glucose fluctuations and both oxidative stress and endothelial cell apoptosis, which may lead to microvascular damage, inflammatory milieu, and disruption of normal immune function. ${ }^{24-26}$. In addition to these potential mechanisms, hyperglycemia is known to 1) impair neutrophil activity including chemotaxis, formation of reactive oxygen species, and phagocytosis; 2) impair lymphocytes by increasing apoptosis, suppressing proliferation and attenuating antibody production and function; and 3) increase pro-inflammatory state cytokines such as IL-6 and TNF- $\alpha^{1,15,20,21,27-29}$ These changes, if true of variability as well, may explain the association between GV and viral infections, particularly through lymphocyte dysfunction. Further, if GV potentiates post-HSCT inflammatory milieu, increased risk for HSCT complications, more generally, may be expected.

Understanding the degree and mechanism by which GV contributes to HSCT complications and, potentially, mortality, and how these might be modifiable with improved glycemic control, is critical. If these are strengthened with future prospective studies, patients may benefit from supportive care interventions (e.g. enteral feeding instead of TPN, selection of immunosuppressive agents, insulin therapy) aimed at reducing GV. It is important to note, that multiple pediatric and adult studies evaluating the benefit of improved glucose control in non-HSCT patients in the intensive care setting were disappointing, often due to high risk of hypoglycemia. ${ }^{30-34}$ However, diabetes technology has progressed since these studies, and safe, intensive glucose control is increasingly feasible. Further, HSCT patients, who are likely to be at risk for longer than typical ICU patients, may find more benefit.

Even if there is no causal link, GV may serve as a useful biomarker for risk of post-HSCT complications such as infection or critical illness; predicting abnormal glucose control based on pre-HSCT characteristics may be useful for tools such as the pre-transplant comorbidity prognostic index. ${ }^{35}$ However, prior to assessing the utility of adjusting such tools or performing glucose interventions in patients, additional prospective work is needed. 
This study has several limitations that must be considered when discussing implications and future studies. As with any retrospective study, causality cannot be determined. Prospective studies are necessary to confirm our results, as well as re-evaluate for associations between GV and mortality. Further, outcomes such as invasive fungal infection and GVHD relied on correct international classification of diseases (ICD) diagnostic coding and manual review and therefore have potential for misclassification bias. While this is unlikely to bias the data as classification challenges would be true across all patients, it may have resulted in decreased statistical power due to underreporting. Most limiting was our previously mentioned inability to evaluate for associations between post-HSCT GV and outcomes that tended to occur early in the post-HSCT period, such as infection and ICU hospitalization. As such, it is possible that this study missed a true underlying association between post-HSCT GV and increased infection or ICU stays. Additionally, while this study aims to highlight correlations between GV and adverse events, any biological causes are hypothetical and require prospective human and animal model-based studies. To this end, future work will use continuous glucose monitoring to prospectively describe GV, its risk factors, and associated outcomes. While this study demonstrated relationships between stress GV immediately preceding and following HSCT and outcomes; future work should evaluate whether GV that predates HSCT-related stress is also associated with adverse outcomes.

In summary, GV is a common occurrence in the pediatric HSCT population and is associated with increased risk of infection and critical illness in HSCT patients. Because GV is subtle and may be more easily overlooked than hyperglycemia or hypoglycemia by care teams, special attention to understanding its incidence, risk factors, and implications, is necessary.

Conflicts of Interest

G.P.F. has served as a consultant for Abbott Diabetes Care, an advisory board member for Dexcom, and conducts research sponsored by Medtronic, Dexcom, Bigfoot, Tandem, Insulet, and NovoNordisk. MRV has served as a consultant for Fate Therapeutics and B-mogen. All other authors declare no competing financial interests.

Acknowledgements

This work was supported by the National Institutes of Health (grant NIDDK 2K12DK094712-06). Contents are the authors' sole responsibility and do not necessarily represent official NIH views.

Data Sharing

The data that support the findings of this study are available from the corresponding author upon reasonable request.

Correspondence: Jenna Sopfe, Center for Cancer and Blood Disorders, Department of Pediatrics, University of Colorado School of Medicine, 13123 E. 16 ${ }^{\text {th }}$ Ave, B115, Aurora, Colorado, 80045; email: jenna.sopfe@cuanschutz.edu

\section{References}

1. Hammer MJ, Casper C, Gooley TA, O'Donnell PV, Boeckh M, Hirsch IB. The contribution of malglycemia to mortality among allogeneic hematopoietic cell transplant recipients. Biol Blood Marrow Transplant. 2009;15(3):344-351.

2. Storey S, Von Ah D. Impact of malglycemia on clinical outcomes in hospitalized patients with cancer: a review of the literature. Oncol Nurs Forum. 2012;39(5):458-465.

3. Sopfe J, Pyle L, Keating AK, et al. Malglycemia is associated with poor outcomes in pediatric and adolescent hematopoietic stem cell transplant patients. Blood Adv. 2019;3(3):350-359.

4. Rodbard D. The challenges of measuring glycemic variability. Journal of diabetes science and technology. 2012;6(3):712-715. 
5. Monnier L, Colette C, Wojtusciszyn A, et al. Toward Defining the Threshold Between Low and High Glucose Variability in Diabetes.Diabetes Care. 2017;40(7):832-838.

6. Kovatchev B, Cobelli C. Glucose Variability: Timing, Risk Analysis, and Relationship to Hypoglycemia in Diabetes. Diabetes care.2016;39(4):502-510.

7. Danne T, Nimri R, Battelino T, et al. International Consensus on Use of Continuous Glucose Monitoring. Diabetes care.2017;40(12):1631-1640.

8. Salkind SJ, Huizenga R, Fonda SJ, Walker MS, Vigersky RA. Glycemic variability in nondiabetic morbidly obese persons: results of an observational study and review of the literature. J Diabetes Sci Technol. 2014;8(5):1042-1047.

9. MacMillan ML, Weisdorf DJ, Wagner JE, et al. Response of 443 patients to steroids as primary therapy for acute graft-versus-host disease: comparison of grading systems. Biol Blood Marrow Transplant.2002;8(7):387394.

10. Socie G, Stone JV, Wingard JR, et al. Long-term survival and late deaths after allogeneic bone marrow transplantation. Late Effects Working Committee of the International Bone Marrow Transplant Registry. $N$ Engl J Med. 1999;341(1):14-21.

11. Cetin M, Yetgin S, Kara A, et al. Hyperglycemia, ketoacidosis and other complications of L-asparaginase in children with acute lymphoblastic leukemia. J Med. 1994;25(3-4):219-229.

12. Yoshida H, Imamura T, Saito AM, et al. Protracted Administration of L-Asparaginase in Maintenance Phase Is the Risk Factor for Hyperglycemia in Older Patients with Pediatric Acute Lymphoblastic Leukemia.PLoS One. 2015;10(8):e0136428.

13. Gatzioura I, Papakonstantinou E, Dimitriadou M, et al. Glucose Levels Before the Onset of Asparaginase Predicts Transient Hyperglycemia in Children With Acute Lymphoblastic Leukemia. Pediatr Blood Cancer. 2016;63(7):1181-1184.

14. Petryk A, Kanakatti Shankar R, Giri N, et al. Endocrine disorders in Fanconi anemia: recommendations for screening and treatment. J Clin Endocrinol Metab. 2015;100(3):803-811.

15. Derr RL, Hsiao VC, Saudek CD. Antecedent hyperglycemia is associated with an increased risk of neutropenic infections during bone marrow transplantation. Diabetes Care. 2008;31(10):1972-1977.

16. Fuji S, Kim SW, Mori S, et al. Hyperglycemia during the neutropenic period is associated with a poor outcome in patients undergoing myeloablative allogeneic hematopoietic stem cell transplantation. Transplantation. 2007;84(7):814-820.

17. Garg R, Bhutani H, Alyea E, Pendergrass M. Hyperglycemia and length of stay in patients hospitalized for bone marrow transplantation.Diabetes Care. 2007;30(4):993-994.

18. Pidala J, Kim J, Kharfan-Dabaja MA, et al. Dysglycemia following glucocorticoid therapy for acute graft-versus-host disease adversely affects transplantation outcomes. Biol Blood Marrow Transplant.2011;17(2):239-248.

19. Sheean $\mathrm{P}$, Braunschweig $\mathrm{C}$. The incidence and impact of dextrose dose on hyperglycemia from parenteral nutrition (PN) exposure in hematopoietic stem cell transplant (HSCT) recipients. JPEN J Parenter Enteral Nutr. 2006;30(4):345-350.

20. de Souza Ferreira C, Araujo TH, Angelo ML, et al. Neutrophil dysfunction induced by hyperglycemia: modulation of myeloperoxidase activity. Cell Biochem Funct. 2012;30(7):604-610.

21. Gyurko R, Siqueira CC, Caldon N, Gao L, Kantarci A, Van Dyke TE. Chronic hyperglycemia predisposes to exaggerated inflammatory response and leukocyte dysfunction in Akita mice. J Immunol.2006;177(10):7250-7256. 
22. Fuji S, Rovo A, Ohashi K, et al. How do I manage hyperglycemia/post-transplant diabetes mellitus after allogeneic HSCT.Bone Marrow Transplant. 2016.

23. Fuji S, Loffler J, Savani BN, Einsele H, Kapp M. Hyperglycemia as a possible risk factor for mold infections-the potential preventative role of intensified glucose control in allogeneic hematopoietic stem cell transplantation. Bone Marrow Transplant. 2017;52(5):657-662.

24. Monnier L, Mas E, Ginet C, et al. Activation of oxidative stress by acute glucose fluctuations compared with sustained chronic hyperglycemia in patients with type 2 diabetes. JAMA. 2006;295(14):1681-1687.

25. Quagliaro L, Piconi L, Assaloni R, Martinelli L, Motz E, Ceriello A. Intermittent high glucose enhances apoptosis related to oxidative stress in human umbilical vein endothelial cells: the role of protein kinase $\mathrm{C}$ and NAD(P)H-oxidase activation. Diabetes. 2003;52(11):2795-2804.

26. Risso A, Mercuri F, Quagliaro L, Damante G, Ceriello A. Intermittent high glucose enhances apoptosis in human umbilical vein endothelial cells in culture. Am J Physiol Endocrinol Metab.2001;281(5):E924-930.

27. Stegenga ME, van der Crabben SN, Blumer RM, et al. Hyperglycemia enhances coagulation and reduces neutrophil degranulation, whereas hyperinsulinemia inhibits fibrinolysis during human endotoxemia.Blood. 2008;112(1):82-89.

28. Oldenborg PA, Sehlin J. Hyperglycemia in vitro attenuates insulin-stimulated chemokinesis in normal human neutrophils. Role of protein kinase C activation. J Leukoc Biol. 1999;65(5):635-640.

29. McCowen KC, Malhotra A, Bistrian BR. Stress-induced hyperglycemia.Crit Care Clin. 2001;17(1):107124.

30. Agus MS, Wypij D, Hirshberg EL, et al. Tight Glycemic Control in Critically Ill Children. The New England journal of medicine.2017;376(8):729-741.

31. Finfer S, Chittock DR, Su SY, et al. Intensive versus conventional glucose control in critically ill patients. The New England journal of medicine. 2009;360(13):1283-1297.

32. Investigators N-SS, Finfer S, Liu B, et al. Hypoglycemia and risk of death in critically ill patients. $N$ Engl J Med.2012;367(12):1108-1118.

33. Agus MS, Asaro LA, Steil GM, et al. Tight glycemic control after pediatric cardiac surgery in high-risk patient populations: a secondary analysis of the safe pediatric euglycemia after cardiac surgery trial.Circulation. 2014;129(22):2297-2304.

34. De Block CE, Gios J, Verheyen N, et al. Randomized Evaluation of Glycemic Control in the Medical Intensive Care Unit Using Real-Time Continuous Glucose Monitoring (REGIMEN Trial). Diabetes Technol Ther. 2015;17(12):889-898.

35. Smith AR, Majhail NS, MacMillan ML, et al. Hematopoietic cell transplantation comorbidity index predicts transplantation outcomes in pediatric patients. Blood. 2011;117(9):2728-2734.

Figure legends

Figure 1. Distribution of patient glucose coefficient of variation (CV) values for time interval and grade. The pre-HSCT interval is shown in Panel A and the day 0-30 post-HSCT interval in Panel B. Glucose CVs are color-coded to demonstrate grades: Grade $1<20 \%$ (green), approximating CV of healthy adults (Grade 1, <20\%); Grade 2 [?]20-<27\% (orange), approximating CV of adults who are obese or receive aggressive oral therapy for type 2 diabetes; Grade 3 [?]27- $<36 \%$ (purple), approximating CV of adults with type 1 or 2 diabetes that is well-controlled with insulin; and Grade 4 [?]36\% (pink), which has been defined as the cutoff for adults with poorly controlled type 1 diabetes. The distribution of patient glucose CVs demonstrates right skew and a marked number of patients with glucose CVs in the range of glucose CVs of adults who are obese or have diabetes. 
Figure 2. Hazard ratios for all-cause mortality by glucosecoefficient of variation (CV) stratified by hematopoietic stem cell transplant (HSCT) type for the pre-HSCT (Panel A) and day 0-30 (Panel B) intervals; hazard ratios for transplant-related mortality (TRM) for the pre-HSCT (Panel C) and day 0-30 (Panel D) intervals. There was an interaction between glucose CV and HSCT type for risk of all-cause mortality models ( $\mathrm{p}=0.079$ pre-HSCT, $\mathrm{p}=0.02$ post-HSCT); as such, Panels A and $\mathrm{B}$ are stratified by HSCT type. Among allogeneic HSCT patients and after adjusting for post-HSCT steroid therapy and diagnosis of severe graft versus host disease (GVHD), there was not a significant association between pre-HSCT glucose CV and risk of death (HR 1.40; 95\% CI 0.98-1.99) (Panel A). In a similar analysis for day 0-30 glucose CV, there was an increase in risk of death with every doubling of day 0-30 glucose CV (HR 1.38; 95\% CI 1.00-1.91) (Panel B). In contrast to risk of all-cause mortality, there was no interaction between glucose CV and HSCT type in TRM models. There was not a significant association between preHSCT (HR 1.42; 95\% CI 0.92-2.19) or day 0-30 (HR 1.33; 95\% CI 0.86-2.06) glucose CV and TRM, after adjusting for HSCT type, post-HSCT steroid therapy, and severe GVHD (Panels C-D).

Figure 3. Adjusted hazard ratio for time-to-infection (top figure) and adjusted odds of infection subtypes (bottom figure) for each doubling of pre-hematopoietic stem cell transplant (HSCT) glucose coefficient of variation (CV). There was a 4.91 (95\% CI 1.4-17.24)-fold higher risk of infection with each doubling of pre-HSCT glucose CV, after adjusting for transplant type. Among allogeneic HSCT patients, there was a 1.77-fold (95\% CI 1.23-2.56) increased odds of viremia/viruria post-HSCT with every doubling of pre-HSCT glucose CV. Odds of viremia was not assessed for autologous HSCT patients. Among all patients, adjusted for HSCT type, there was no increased odds of serious bacterial illness (SBI) or fungal infection with increasing pre-HSCT glucose CV.

\section{Hosted file}

TABLE 1 Patient Characteristics.docx available at https://authorea.com/users/321737/articles/ 450941-glycemic-variability-is-associated-with-poor-outcomes-in-pediatric-hematopoieticstem-cell-transplant-patients

\section{Hosted file}

TABLE 2 Multivariable model of glucose CV and potential risk factors.docx available at https://authorea.com/users/321737/articles/450941-glycemic-variability-is-associatedwith-poor-outcomes-in-pediatric-hematopoietic-stem-cell-transplant-patients

\section{Hosted file}

TABLE 3. Adjusted hazard ratios for adverse outcomes with each doubling of glucose coefficient of varia available at https://authorea.com/users/321737/articles/450941-glycemic-variability-isassociated-with-poor-outcomes-in-pediatric-hematopoietic-stem-cell-transplant-patients 

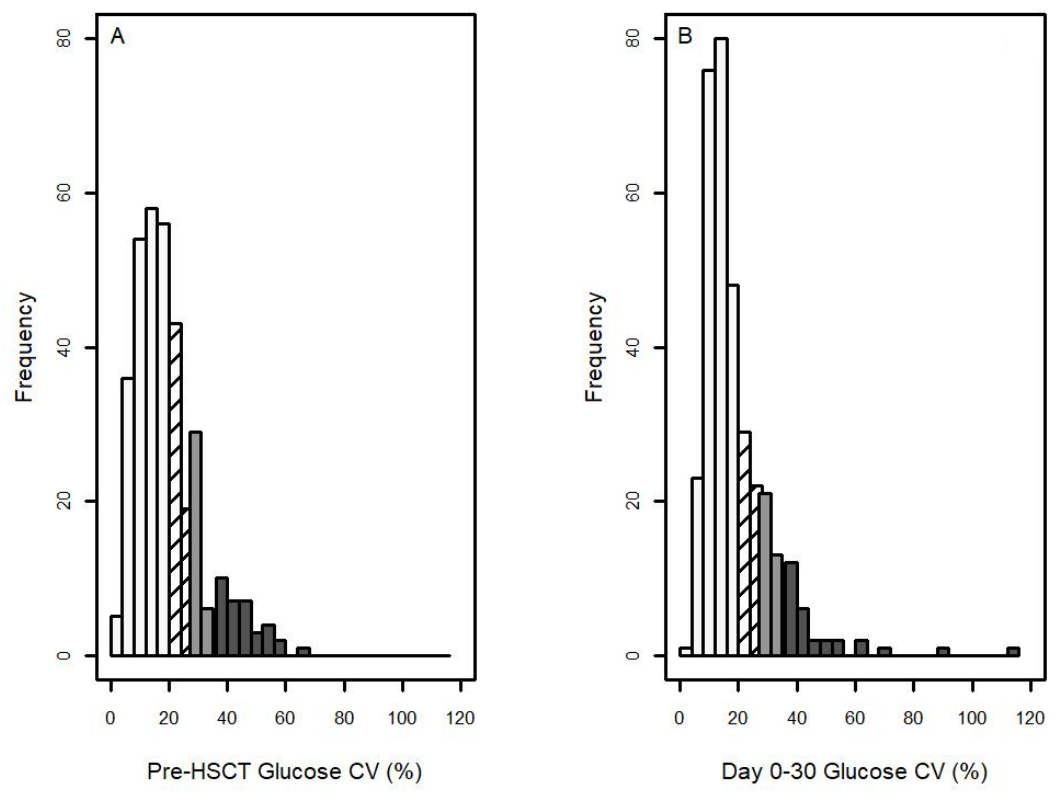

Legend:

$\square$ Grade $1:<20 \% \quad \square \quad$ Grade 2: 20-27\% $\square$ Grade 3: $27-36 \% \quad \square \quad$ Grade 4:36\%+ 

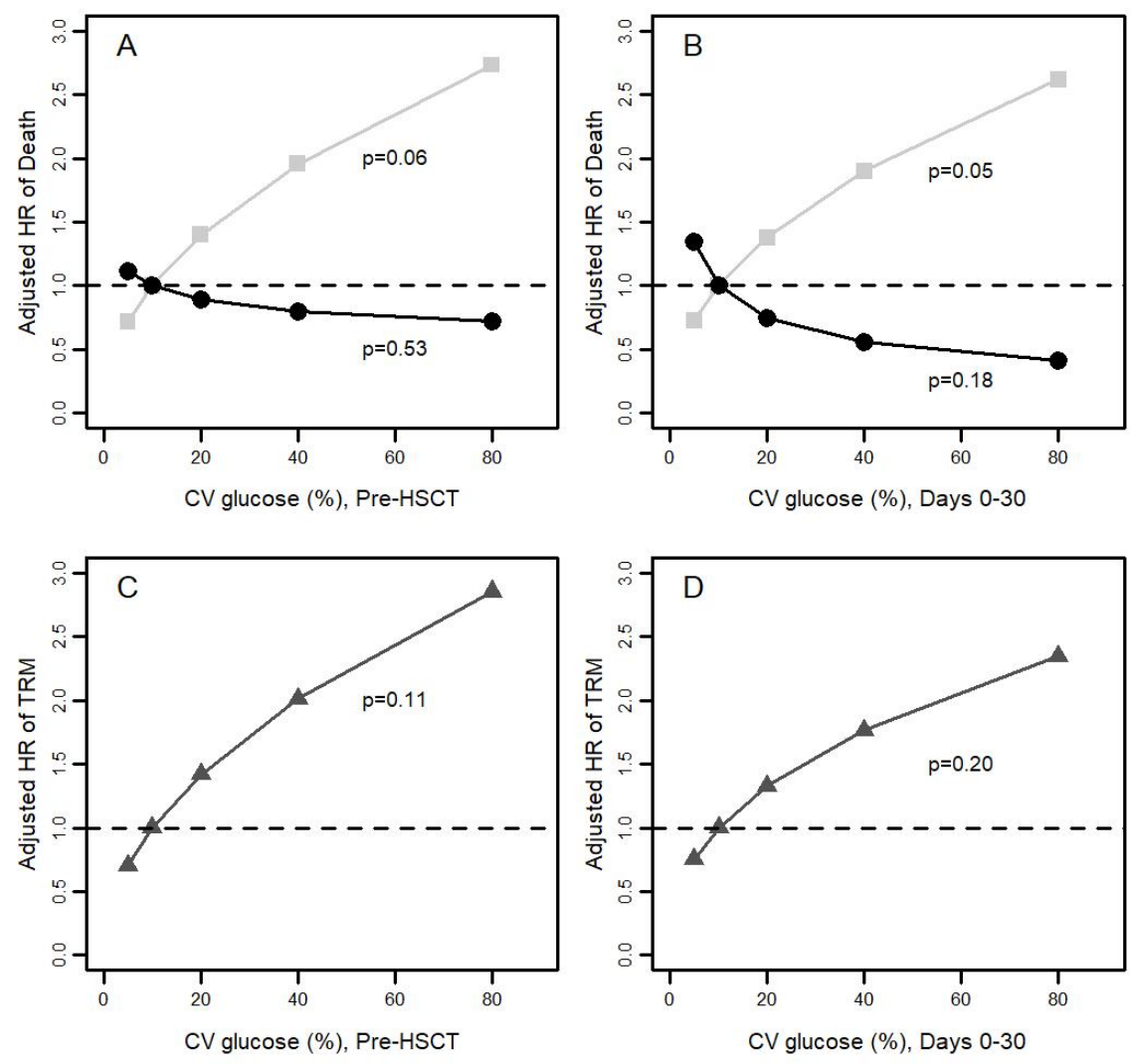

Legend:

Allogenic $\longrightarrow$ Autologous $\longrightarrow$ 

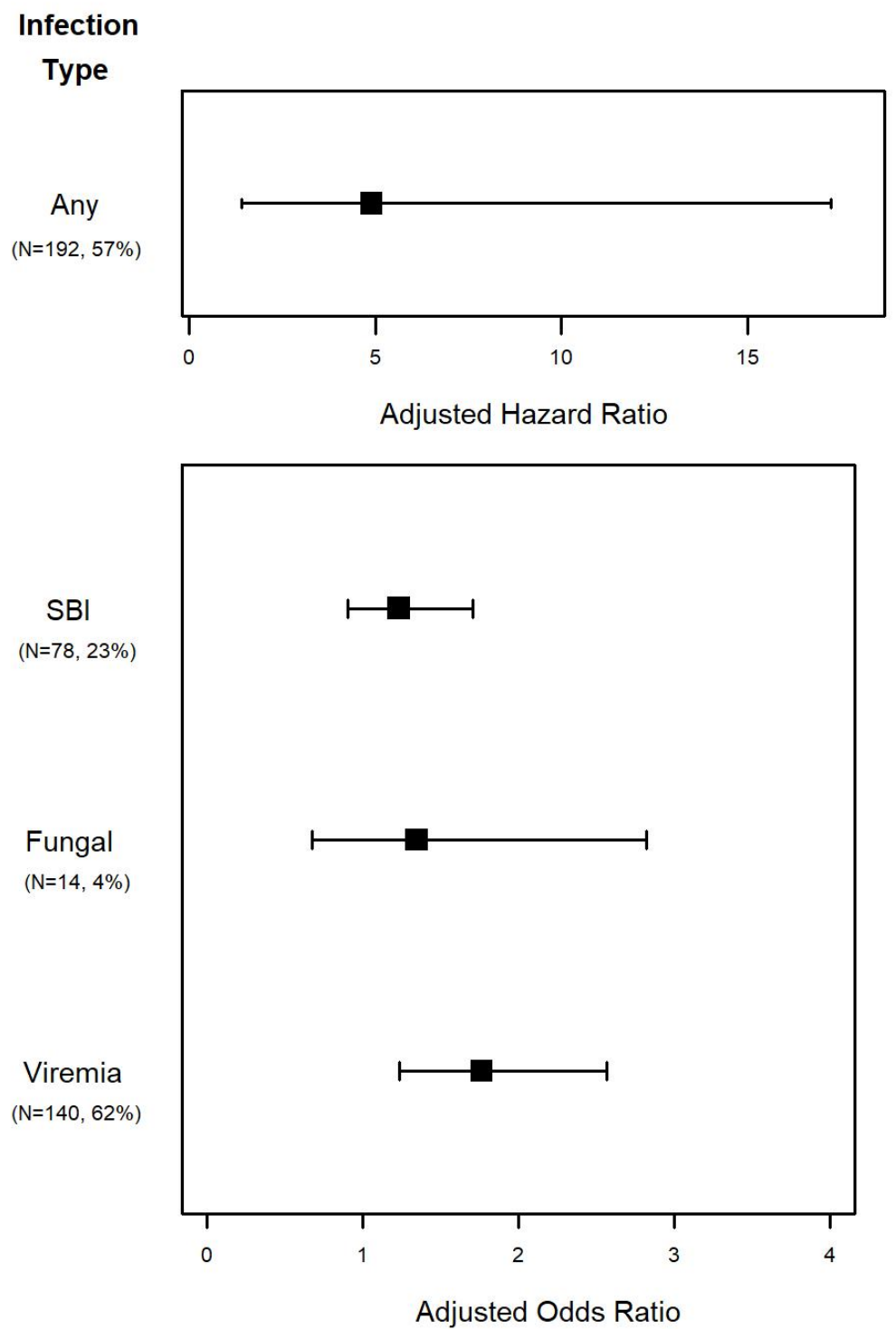